\title{
Endothelial-Specific Overexpression of Caveolin-1 Accelerates Atherosclerosis in Apolipoprotein E-Deficient Mice
}

\author{
Carlos Fernández-Hernando, ${ }^{* \dagger}$ Jun $\mathrm{Yu},{ }^{*}$ \\ Alberto Dávalos, ${ }^{*}$ Jay Prendergast, ${ }^{*}$ \\ and William C. Sessa*
}

From the Department of Pharmacology and Vascular Biology and Therapeutics Program," Yale University School of Medicine, New Haven, Connecticut; and the Department of Medicine, Leon H. Charney Division of Cardiology, and Cell Biology and the Marc and Ruti Bell Vascular Biology and Disease Program, ${ }^{\dagger}$ New York University School of Medicine, New York, New York

Caveolin-1 (Cav-1) is the major structural protein essential to the formation of the caveolae in endothelial cells. Genetic ablation of Cav-1 on an apolipoprotein E knockout background inhibits the progression of atherosclerosis, whereas re-expression of Cav-1 in the endothelium promotes lesion expansion. Although Cav1-null mice are useful to delineate the importance of caveolae in atherosclerosis, there are additional problems that are difficult to dissect because loss of Cav-1 abolishes both the caveolae organelle as well as the Cav-1-mediated signaling pathways. To study how Cav-1 influences the progression of atherosclerosis in mice with caveolae, we generated a transgenic mouse that overexpresses Cav-1 in the endothelial cells in an apolipoprotein E-deficient background. We found that endothelial-specific overexpression of Cav-1 enhanced the progression of atherosclerosis in mice. Mechanistically, overexpression of Cav-1 reduced endothelial cell proliferation, migration, and nitric oxide production in vitro and increased expression of vascular cell adhesion molecule-1 in vivo. (Am J Pathol 2010, 177:998-1003; DOI: 10.2353/ajpath.2010.091287)

Atherosclerosis is a progressive disease characterized by the accumulation of lipids, inflammatory cells, and fibrous elements in the large arteries. ${ }^{1,2}$ In Western societies, it is the underlying cause of approximately $50 \%$ of all deaths. ${ }^{2}$ Traditional hypotheses of atherogenesis have suggested that the dysfunction or injury of vascular endothelial cells (ECs) is critical for the development of atherosclerosis. ${ }^{3,4}$ The endothelium functions as a selectively permeable barrier between blood and tissues as it can regulate transcytosis and generate effector molecules such us nitric oxide (NO) that regulate thrombosis, inflammation, vascular tone, and vascular remodeling. ${ }^{5}$ The anatomic sites where the atheromas develop are associated with perturbed flow and increased EC turnover rate and these athero-prone areas show increased permeability to macromolecules such as low-density lipoproteins (LDLs) at preferential sites for lesion formation. ${ }^{6}$

Caveolae are 50- to 100-nm cell surface flask-shaped invaginated structures observed in many cell types including endothelium. ${ }^{7}$ Among the three isoforms of caveolin (Cav) that have been described (Cav-1, Cav-2, and Cav3), Cav-1 is the major coat protein of endothelial caveolae and is necessary for caveolae assembly. ${ }^{8,9}$ Indeed, several studies have shown a complete loss of caveolae organelles in blood vessels, adipocytes, and fibroblasts obtained from Cav-1-deficient mice. ${ }^{8,9}$ The putative functions of caveolae include cholesterol transport, endocytosis, transcytosis, and signal transduction. Physiologically, an absence of caveolae results in impairment of cholesterol homeostasis, insulin resistance, nitric oxide production, and calcium signaling..$^{10-12}$ In the cardiovascular system, caveolae and Cav-1 show an important role in the proliferation and migration of smooth muscle cells, ${ }^{13}$ regulation of NO production and vascular permeability, ${ }^{8,14}$ angiogenesis, ${ }^{15-17}$ and atherosclerosis. ${ }^{18,19}$ Cav-1-deficient mice develop cardiac hypertrophy, hypercholesterolemia, and hypertriglyceridemia and have increased tumor microvascular permeability, angiogenesis, and growth. ${ }^{17}$ Interestingly, the absence of Cav-1 reduces the progression of atherosclerosis. ${ }^{19}$ Very recently, we showed, using a genetic model, that endo-

Supported by the National Institutes of Health (grants R01 HL64793, R01 HL61371, R01 HL081190, and P01 HL70295 to W.C.S.) and the American Heart Association (Scientist Development grant to C.F.-H. and J.Y.). A.D. is the recipient of a postdoctoral fellowship from Instituto de Salud Carlos III, Spain.

Accepted for publication March 30, 2010.

Address reprint requests to William C. Sessa, Ph.D., Amistad Building, 10 Amistad St., New Haven, CT 06520. E-mail: william.sessa@yale.edu. 
thelial caveolae are essential for the progression of atherosclerosis. ${ }^{18}$ Mechanistically, endothelial Cav-1 and caveolae are critical for LDL infiltration in the artery wall, NO production, and macrophage accumulation, all events necessary for atherogenesis. ${ }^{18}$ Although Cav-1null mice are useful to delineate the importance of caveolae in atherosclerosis, there are additional problems that are difficult to dissect, because loss of Cav-1 abolishes both caveolae organelles as well as Cav-1-mediated signaling pathways. To study how Cav-1 influences the progression of atherosclerosis in mice with caveolae, we generated a transgenic mice that overexpress Cav- 1 in the endothelial cells in an apolipoprotein E-deficient $\left(A p o E^{-/-}\right)$background. We found that endothelial-specific overexpression of Cav-1 enhanced the progression of atherosclerosis in mice. Mechanistically, overexpression of Cav-1 reduces EC proliferation, migration, and NO production and increases vascular cell adhesion molecule 1 (VCAM-1) expression in vivo.

\section{Materials and Methods}

\section{Animal Procedures}

Endothelial-specific Cav-1 ${ }^{T G}$ heterozygous mice carrying a canine Cav-1 transgene under the preproendothelin-1 promoter were crossed six generations with F6 generation $A p o E^{-\prime-}$, as reported previously. ${ }^{20}$ Accelerated atherosclerosis was induced by feeding the mice for 14 weeks with a high-fat Western-type diet containing 1.25\% cholesterol (D12108, Research Diets, New Brunswick, $\mathrm{NJ}$ ). The Institutional Animal Care Use Committee of Yale University approved all of the experiments.

\section{Atherosclerotic Lesion Analysis}

After 12 weeks of a Western-type diet, mice were anesthetized and euthanized. Hearts were perfused using 10 $\mathrm{ml}$ of PBS (Invitrogen, Carlsbad, CA) followed by $10 \mathrm{ml}$ of $4 \%$ paraformaldehyde. After incubation in $4 \%$ paraformaldehyde overnight, the adventitia was thoroughly cleaned under a dissecting microscope, and the aorta was cut open longitudinally and pinned onto a silicone plate. To calculate the lesion area, aortas were stained with Oil Red O (Sigma-Aldrich, St. Louis, MO) before the analysis. Oil Red O stock solution $(35 \mathrm{ml} ; 0.2 \% \mathrm{w} / \mathrm{v}$ in methanol) was mixed with $10 \mathrm{ml}$ of $1 \mathrm{~mol} / \mathrm{L} \mathrm{NaOH}$ and filtered. Aortas were briefly rinsed with $78 \%$ methanol, incubated in Oil Red O solution for 50 minutes, and then destained in $78 \%$ methanol for 5 minutes and mounted on microscopic slides using aqueous mounting medium (Stephens Scientific, Wayne, NJ). Plaques were analyzed with a Nikon SMZ 1000 microscope connected to a Kodak DC290 digital camera. The images were analyzed using Adobe Photoshop 6.0 (Adobe Systems, Mountain View, CA), and the lesions were quantified using ImageJ $(\mathrm{NIH}$, Bethesda, MD). For other images, 10- $\mu \mathrm{m}$-thick cryosections of the proximal aorta were serially sectioned and stained with H\&E for quantification of the lesion areas using ImageJ. The aortic lesion size of each animal was obtained by averaging lesion areas in six sections from the same mouse.

\section{Western Blot Analysis}

Tissues were snap-frozen in liquid nitrogen, pulverized, and resuspended in lysis buffer: $50 \mathrm{mmol} / \mathrm{L}$ Tris- $\mathrm{HCl}, \mathrm{pH}$ 7.4, $0.1 \mathrm{mmol} / \mathrm{L}$ EDTA, $0.1 \mathrm{mmol} / \mathrm{L}$ EGTA, $1 \%$ Nonidet P-40, $0.1 \%$ sodium deoxycholate, $0.1 \%$ SDS, $100 \mathrm{mmol} / \mathrm{L}$ $\mathrm{NaCl}, 10 \mathrm{mmol} / \mathrm{L} \mathrm{NaF}, 1 \mathrm{mmol} / \mathrm{L}$ sodium pyrophosphate, $1 \mathrm{mmol} / \mathrm{L}$ sodium orthovanadate, $1 \mathrm{mmol} / \mathrm{L}$ Pefabloc SC, and $2 \mathrm{mg} / \mathrm{ml}$ protease inhibitor cocktail (Roche Diagnostics, Indianapolis, IN). Cells were lysed on ice with lysis buffer as noted above. Protein concentrations were determined using the DC Protein Assay Kit (Bio-Rad Laboratories, Hercules, CA). Lysates containing $80 \mu \mathrm{g}$ (tissue) or $30 \mu \mathrm{g}$ (cells) of protein were analyzed by SDS-polyacrylamide gel electrophoresis and immunoblotting. Primary antibodies used include the following: Akt (Cell Signaling Technology, Danvers, MA), endothelial NO synthase (eNOS), Cav-1, Cav-2, heat shock protein (HSP90) (BD Transduction Laboratories, BD Biosciences Pharmingen, San Diego, CA), VCAM-1 and intercellular adhesion molecule 1 (ICAM-1) (R\&D Systems, Minneapolis, MN), and $\beta$-actin (Sigma-Aldrich). Secondary antibodies were fluorescence-labeled antibodies (LI-COR Biotechnology, Lincoln, NB). Bands were visualized using the Odyssey Infrared Imaging System (LI-COR Biotechnology).

\section{Lipid Analysis and Lipoprotein Profile Measurement}

Mice were fasted for 12 to 14 hours before blood samples were collected by retro-orbital venous plexus puncture. Plasma was separated by centrifugation and stored at $-80^{\circ} \mathrm{C}$. Total plasma cholesterol and triglycerides were enzymatically measured using the Amplex red cholesterol assay kit (Molecular Probes, Eugene, OR) and a serum triglyceride determination kit (Sigma-Aldrich), respectively, according to the manufacturer's instructions. The lipid distribution in plasma lipoprotein fractions was assessed by fast-performance liquid chromatography gel filtration with two Superose 6 HR 10/30 columns (Pharmacia, Peapack, NJ).

\section{Mouse Lung Endothelial Cell Isolation}

Mouse lung endothelial cells (MLECs) were isolated from 3-week-old wild-type and Cav-1 ${ }^{T G}$ mice. In brief, mice were euthanized with an overdose of ketamine/xylazine, and the lungs were excised, minced, and digested with $0.1 \%$ collagenase in RPMI 1640 medium. The digest was homogenized by passing multiple times through a 14gauge needle. It was then filtered through a $150-\mu \mathrm{m}$ tissue sieve, and the cell suspension was plated on $0.1 \%$ gelatin-coated dishes. After 2 to 3 days, cells were immortalized by two rounds of infection with retrovirus encoding the middle $T$ antigen. Cells were allowed to recover for 24 hours, and then ECs were isolated by 
immunoselection with platelet/endothelial cell adhesion molecule 1- and ICAM-2-conjugated magnetic beads. When cells reached confluence, a second round of immunoselection was performed. Cells were propagated in EGM-2 medium supplemented with 15\% fetal bovine serum (FBS) and EGM-2 microvascular SingleQuots (Cambrex, East Rutherford, NJ).

\section{MLEC Proliferation Assay}

MLECs were grown in EGM-2 medium supplemented with 15\% FBS and EGM-2 microvascular SingleQuots. At the indicated times, the viable cells were determined by Trypan blue dye exclusion using a hemocytometer.

\section{MLEC Migration Assay}

Cell migration assays were performed with a modified Boyden chamber with Costar Transwell inserts (Corning, Corning, NY). The Transwell inserts were coated with $0.1 \%$ gelatin (Sigma-Aldrich). MLECs $\left(5 \times 10^{4}\right.$ cells per well) were starved overnight, resuspended in $100 \mathrm{ml}$ of EBM-2 medium containing $0.1 \% \mathrm{FBS}$, and added to the upper chamber. EBM-2 medium containing $0.1 \%$ FBS or complete media was added in the bottom chamber. After 5 hours of incubation, cells on both sides of the membrane were fixed and stained with a Diff-Quick staining kit (Baxter Healthcare Corp, Deerfield, IL). Cells on the upper side of the membrane were removed with a cotton swab. The average number of cells from five randomly chosen high-power $(\times 200)$ fields on the lower side of the membrane was counted.

\section{NO Release Analysis}

MLECs were incubated in the absence of serum for 8 hours, and then the medium was removed and the cells were supplemented with serum-free EGM-2 medium for 12 hours. The medium was then processed for the measurement of nitrite $\left(\mathrm{NO}_{2}^{-}\right)$by an NO-specific chemiluminescence analyzer (Sievers, Boulder, $\mathrm{CO}$ ) as described previously. ${ }^{21}$

\section{Statistical Analysis}

Data are presented as means \pm SE $(n$ is noted in the figure legends). Comparison of mean values between groups was evaluated by a two-tailed Student's $t$-test and Mann-Whitney $U$-test. $P<0.05$ was considered significant.

\section{Results}

\section{Endothelial-Specific Overexpression Enhances Atherosclerosis in $\mathrm{ApoE}^{-/-}$Mice}

Cav-1 ${ }^{T G}$ mice were generated by transgenic overexpression of Cav-1 under control of the preproendothelin-1 promoter (Figure 1A). ${ }^{22}$ To assess the effect of endothelial-specific Cav-1 overexpression on the progression of
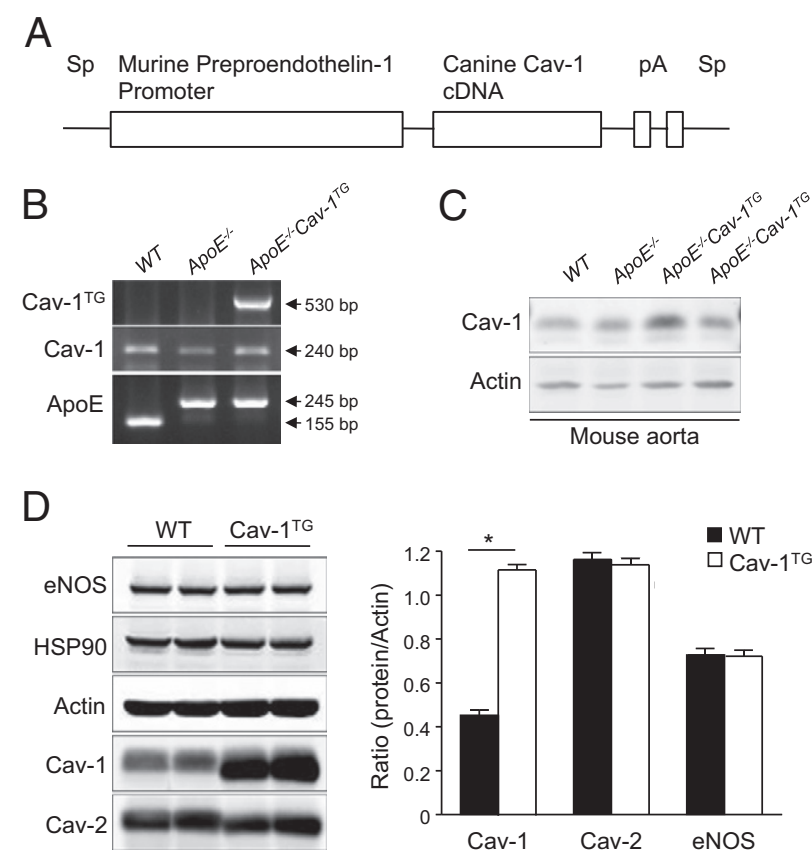

Figure 1. Characterization of Cav-1 endothelial-specific transgenic mice. A: Construct used for generating the Cav-1 transgenic mice. B: Expression of the transgene, endogenous Cav-1, and ApoE alleles. C: Cav-1 protein levels from mouse aorta. D: Western blot analyses (left panel) and densitometry (right panel) of Cav-1, Cav-2, actin, HSP90, and eNOS in lung endothelial cell extract prepared from wild-type (WT), $A p o E^{-/-}$, and $A p o E^{-/-} \mathrm{Cav}-1^{T G}$ mice. Results for two representative mice are shown for each genotype.

atherosclerosis, we bred these mice with the atheroprone mouse model (ApoE knockout mouse). The expression of canine Cav-1 was determined by RT-PCR (Figure 1B) and Western blot (Figure 1C). Western blot analysis revealed that Cav- 1 is twofold overexpressed in aorta of the Cav-1 ${ }^{T G}$ mice compared with wild-type mice (Figure 1C). To determine the expression level of Cav-1 in the endothelial-specific transgenic mice, we isolated lung ECs. As shown in Figure 1D, quantified in the right panel, ECs from endothelial-specific transgenic Cav-1 mice expressed 3 times more than those from Cav-1 wild-type mice. In both groups of mice, expressions of HSP90, actin, eNOS, and Cav-2 were equal (Figure 1D).

To study the functional role of endothelial-specific Cav-1 overexpression during the progression of atherosclerosis, ApoE ${ }^{-/-}$Cav-1 ${ }^{T G}$ and corresponding $A p o E^{-/-}$ mice were fed a high-cholesterol diet for 14 weeks, and aortas were opened longitudinally and stained with Oil Red $O$ to visualize lipid-rich, atherosclerotic plaques. As shown in Figure 2, quantified in the right panels, Cav-1 overexpression increased the number and size of aortic plaques. Similar results were found in males (Figure 2, upper panel) and females (Figure 2, bottom panel).

\section{Similar Body Weight, Cholesterol, Triglyceride, and Lipoprotein Profiles}

Next, we examined multiple metabolic parameters of the mice. Body weights, plasma triglycerides, cholesterol levels, and lipoprotein profiles were similar in both groups before and after feeding of a Western diet (Figure 3, 

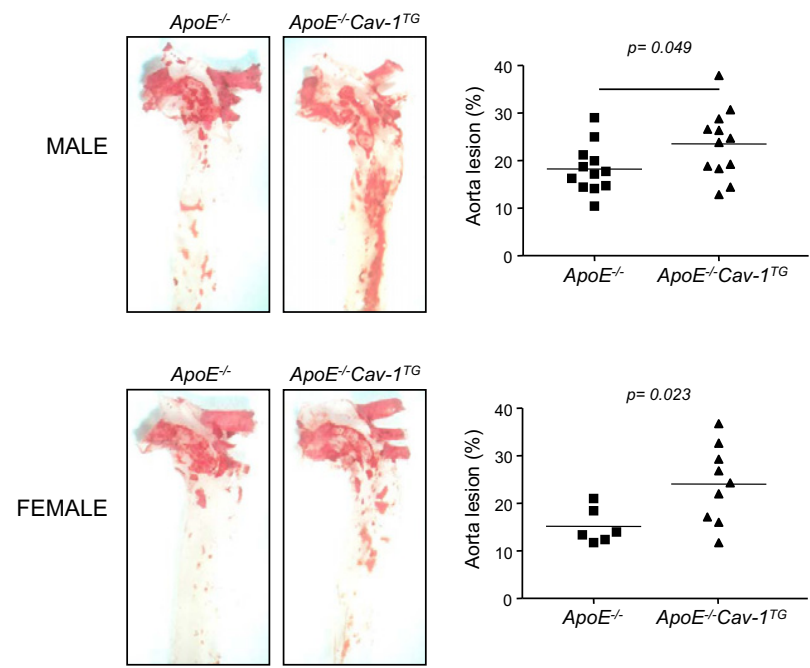

Figure 2. Endothelial-specific overexpression of Cav-1 enhances the progression of atherosclerosis. Oil Red O staining of aortas from mice with the indicated phenotypes. Atheroma formation was significantly increased in $A p o E^{-/-}$Cav $-1^{T G}$ mice $(n=12)$ compared with $A p o E^{-/-}$mice $(n=12)$. Similar results were observed in females $\left[A p o E^{-/} \mathrm{Cav}_{-} 1^{T G}\right.$ mice $(n=9)$; $A p o E^{-/-}$mice $\left.(n=6)\right]$.

A-D), indicating that the increase in lesion formation occurred independently of the plasma lipid profiles in the ApoE ${ }^{-/-}$Cav-1 ${ }^{T G}$ mice.

\section{Endothelial-Specific Cav-1 Overexpression Increases the Expression of VCAM-1 in the Vessel Wall}

Previous reports have showed that the reduced atherosclerosis observed in mice lacking Cav-1 was correlated with reduced levels of VCAM-1 in the artery wall. ${ }^{18,19}$ VCAM-1 is expressed after activation of endothelial cells and mediates the adhesion of leukocytes. As seen in Figure 4, quantified in the right panel, the expression levels of VCAM-1 were significantly up-regulated in the Cav-1 ${ }^{T G}$ mice compared with those in the $A p o E^{-/-}$alone
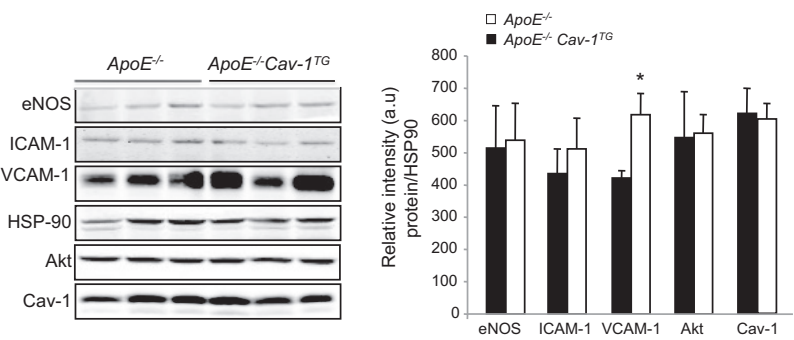

Figure 4. Endothelial-specific overexpression of Cav-1 increases VCAM-1 expression in the artery wall. Western blot analyses (left panel) and densitometry (right panel) of eNOS, ICAM-1, VCAM-1, Akt, and HSP9O in aortic extracts prepared from $A p o E^{-/-}$and $A p o E^{-/} C a v-1^{T G}$ mice fed with a high-cholesterol diet for 12 weeks. Results from three representative mice are shown for each genotype. The data represent the mean \pm SEM of triplicate samples. ${ }^{*} P<0.05$ compared with $A p o E^{-1-}$.

mice. The levels of eNOS, ICAM-1, and Akt were similar in both groups of mice (Figure 4). In contrast to normal arteries (Figure 1C), Cav-1 expression levels were similar in $A p o E^{-/-}$Cav-1 $1^{T G}$ and $A p o E^{-/-}$mice after feeding with a high-cholesterol diet for 14 weeks (Figure 4). Because ApoE ${ }^{-1-}$ Cav-1 $1^{T G}$ mice develop more atherosclerotic plaques, it is possible that the differences in cellular composition in the whole aortas may explain the similar Cav-1 expression levels observed in both groups of mice after a high-cholesterol diet.

\section{Endothelial Cav-1 Overexpression Decreased Endothelial Cell Proliferation and NO Production}

To determine the potential mechanisms of enhanced atherosclerosis in the Cav-1 transgenic mice, we analyzed proliferation, migration, and NO production. Endothelial dysfunction is widely recognized as one of the early alterations in the vessel wall preceding the development of the plaque. In the presence of cardiovascular risk factors endothelial cells are continuously activated and contribute to disease pathogenesis. ${ }^{4}$ To investigate whether Cav-1 overexpression is important for regulating EC functions, we analyzed EC proliferation at different
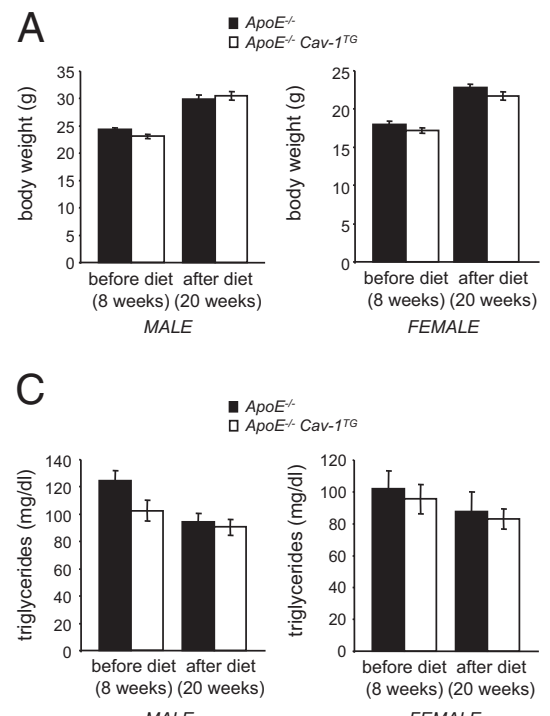

MALE

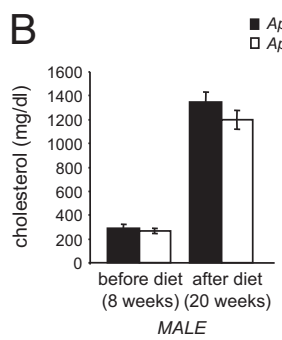

ApoE

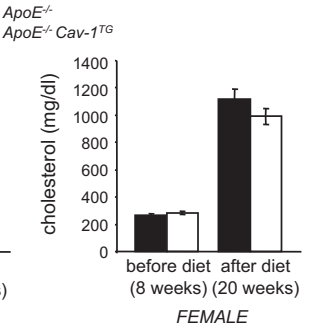

D
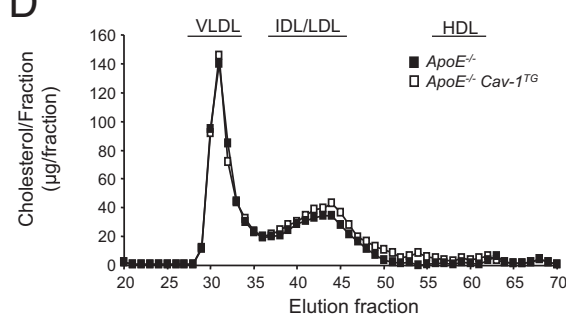

Figure 3. Endothelial-specific overexpression of Cav-1 does not influence body weight, cholesterol, triglycerides, and lipoprotein profiles. A: Body weight of $A p o E^{-1-}(n=12)$ and $A p o E^{-/-}$ Cav- $1^{T G}(n=12)$ mice before and after 12 weeks of being fed a high-cholesterol diet. B: Fasting cholesterol levels of $A p o E^{-/-}(n=12)$ and $A p o E^{-/} \operatorname{Cav} 1^{T G}(n=12)$ mice before and after 12 weeks of being fed a high-cholesterol diet. C: Fasting triglyceride levels from both groups of mice before and after 12 weeks of being fed a high-cholesterol diet. D: Lipoprotein profiles from $A p o E^{-1-}$ and $A p o E^{-1-} C a v-1^{T G}$. VLDL, very low-density cholesterol; IDL, intermediate-density cholesterol; LDL, low-density cholesterol HDL, high-density cholesterol. 
A

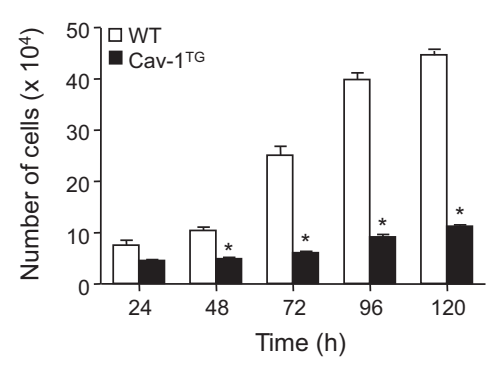

B

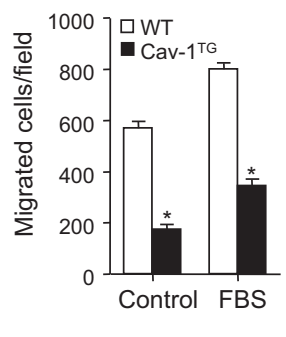

C

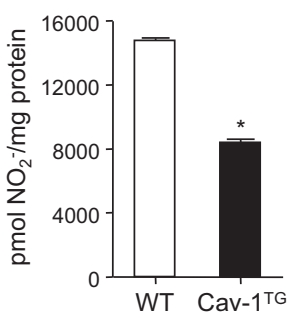

Figure 5. Impaired cell proliferation, migration, and NO release in Cav-1 ${ }^{T C}$ ECs. A: EC proliferation measured as viable cell number at different time points. Proliferation of $C a v-1^{T G}$ ECs was reduced. The data represent the mean \pm SEM of three independent experiments. ${ }^{*} P<0.05$ compared with wild-type (WT) ECs. B: Migration of MLECs was examined in Transwells using FBS as a chemoattractant. Migration of $\mathrm{Cav}-1^{T G}$ ECs was reduced compared with that of wild-type ECs. The data represent the mean \pm SEM of three separate experiments. ${ }^{*} P<0.05$ compared with ECs. C: Basal production of $\mathrm{NO}$ in ECs measured as $\mathrm{NO}_{2}^{-}$in the medium over a 24-hour period was determined by chemiluminescence. Cav-1 overexpression reduces $\mathrm{NO}_{2}^{-}$ accumulation in the medium. The data represent the mean \pm SEM of three separate experiments. ${ }^{*} P<0.05$ compared with ECs. Representative Western blot analyses (right panel) of Cav-1, actin, eNOS, and phospho (p)-eNOS from cell lysates of compared with and $\mathrm{Cav}-1^{T G}$ MLECs used in the NO release experiments.

time points and migration using a transwell assay. As shown in Figure 5A, ECs isolated from Cav-1 ${ }^{T G}$ mice did not grow as well as cells isolated from wild-type mice. A similar result was found when we analyzed cell migration (Figure 5B).

The production of $\mathrm{NO}$ by vascular endothelium is important for cardiovascular homeostasis, as endogenous NO regulates many fundamental processes ${ }^{5}$ and impaired NO bioavailability is a commonly used index of endothelial dysfunction in atherosclerosis. ${ }^{3}$ Genetic deletion of eNOS causes many cardiovascular phenotypes, including accelerated atherosclerosis. ${ }^{23}$ Because endogenous eNOS activation is under the tonic inhibitory influence of Cav-1 as a negative regulator of eNOS activity, ${ }^{24}$ we directly examined the effect of Cav-1 on NO production, using ECs from wild-type and Cav-1 ${ }^{T G}$ mice. As seen in Figure 5C, Cav-1 overexpression reduces NO production, whereas the protein expression levels of eNOS and p-eNOS (right panel) were the same as those in wild-type cells. Thus, reduced NO production by the ECs may also contribute to the increased atherosclerosis observed in the ApoE ${ }^{-/-} \mathrm{Cav}-1^{T G}$ mice. In agreement with that result, we previously reported that endothelial-specific overexpression of Cav-1 impairs eNOS-dependent vasodilatation. $^{22}$

\section{Discussion}

The major finding of this study is that endothelial-specific overexpression of Cav-1 in mice enhances the progression of atherosclerosis. Mechanistically, this phenotype is due to reduced EC proliferation and migration and NO production, leading to enhanced inflammation. Previous studies using the global Cav-1 knockout mouse showed the importance of Cav-1 and caveolae during atherogenesis, ${ }^{18,19}$ and reconstitution of Cav-1 into the endothelium of the global Cav-1-deficient mice rescued this phenotype. ${ }^{18}$ However, the present data combined with the two previous studies suggest that Cav-1, per se, has the capacity to promote atherogenesis in vessels replete with caveolae, perhaps via its scaffolding function.

The role of Cav-1 during the progression of atherosclerosis is complex and depends on the cell type examined. ${ }^{25}$ In vascular smooth muscle cells, the ability of Cav-1 to negatively regulate cell proliferation and migration (neointimal hyperplasia) may have an antiatherogenic effect. ${ }^{13}$ The role of Cav-1 in macrophages remains poorly understood, but several reports have showed that Cav-1 may regulate cholesterol metabolism, phagocytosis, and interaction with high-density lipoproteins. ${ }^{11}$ Cav-1 is highly expressed in endothelial cells and plays an important role in the regulation of the exchange between the blood and the peripheral tissue. ${ }^{9}$ The infiltration and accumulation of LDL in the artery wall is the primary event that leads to atheroma formation. ${ }^{1}$ Very recently, we showed, using a genetic model, that endothelial caveolae are essential for LDL infiltration in the artery wall and the progression of atherosclerosis. ${ }^{18}$ Even though we demonstrated that endothelial caveolae are necessary for LDL transport across the arterial wall in vivo, the mechanisms still remain unclear. Several models have been proposed, including receptor-mediated transcytosis, fluid-phase transcytosis, and paracellular transport. ${ }^{25}$ Another important function of Cav-1 in endothelial cells is its ability to regulate $\mathrm{NO}$ release by inhibition of eNOS activity. ${ }^{24}$ In agreement with that result, we showed that endothelial Cav-1 overexpression has an inhibitory effect on $\mathrm{NO}$ release in endothelial cells. These data support a previous report showing that increasing levels of Cav-1 in the endothelium impair eNOS activation, endothelial barrier function, and angiogenic responses to VEGF without changing endothelial caveolae numbers in vivo. 22

One of the earliest inflammatory events in atherogenesis involves adhesion of leukocytes to the surface of the endothelium. $^{26}$ In particular, VCAM-1 expression is thought to play an important role in the recruitment of monocytes into the artery wall. ${ }^{26}$ Previous studies have shown that increased eNOS activity can down-regulate VCAM-1 expression and, therefore, may reduce monocyte adhesion to the endothelium. ${ }^{27}$ Consistent with this finding, mice lacking Cav-1 expression have increased $\mathrm{NO}$ production and reduced VCAM-1 expression levels in the aorta. ${ }^{8,18,19}$ Interestingly re-expression of Cav-1 in the aortic endothelium rescues the expression of VCAM-1. ${ }^{18}$ Here, we show that endothelial overexpression of Cav-1 also enhanced the expression of VCAM-1 in aortas de- 
rived from Cav-1 transgenic mice, which correlates with the reduced NO production observed in the Cav-1 transgenic mice. ${ }^{22}$

Cav-1 expression has been shown to regulate cell proliferation. ${ }^{28}$ Indeed, mice lacking Cav-1 show a hyperproliferative phenotype in several tissues. ${ }^{9}$ Here, we showed that overexpression of Cav-1 in endothelial cells reduces cell proliferation and migration, which may influence the phenotype observed in vivo. In summary, the findings presented here provide evidence that increasing Cav-1 expression levels, per se, enhance the progression of atherosclerosis.

\section{References}

1. Glass CK, Witztum JL: Atherosclerosis. The road ahead. Cell 2001, 104:503-516

2. Lusis AJ: Atherosclerosis. Nature 2000, 407:233-241

3. Busse R, Fleming I: Endothelial dysfunction in atherosclerosis. J Vasc Res 1996, 33:181-194

4. Dimmeler S, Hermann C, Zeiher AM: Apoptosis of endothelial cells. Contribution to the pathophysiology of atherosclerosis? Eur Cytokine Netw 1998, 9:697-698

5. Sessa WC: eNOS at a glance. J Cell Sci 2004, 117:2427-2429

6. Tricot O, Mallat Z, Heymes C, Belmin J, Leseche G, Tedgui A: Relation between endothelial cell apoptosis and blood flow direction in human atherosclerotic plaques. Circulation 2000, 101:2450-2453

7. Liu P, Rudick M, Anderson RG: Multiple functions of caveolin-1. J Biol Chem 2002, 277:41295-41298

8. Drab M, Verkade P, Elger M, Kasper M, Lohn M, Lauterbach B, Menne J, Lindschau C, Mende F, Luft FC, Schedl A, Haller H, Kurzchalia TV: Loss of caveolae, vascular dysfunction, and pulmonary defects in caveolin-1 gene-disrupted mice. Science 2001, 293:2449-2452

9. Razani B, Engelman JA, Wang XB, Schubert W, Zhang XL, Marks CB, Macaluso F, Russell RG, Li M, Pestell RG, Di Vizio D, Hou H Jr, Kneitz B, Lagaud G, Christ GJ, Edelmann W, Lisanti MP: Caveolin-1 null mice are viable but show evidence of hyperproliferative and vascular abnormalities. J Biol Chem 2001, 276:38121-38138

10. Cohen AW, Razani B, Wang XB, Combs TP, Williams TM, Scherer PE, Lisanti MP: Caveolin-1-deficient mice show insulin resistance and defective insulin receptor protein expression in adipose tissue. Am J Physiol Cell Physiol 2003, 285:C222-C235

11. Frank PG, Cheung MW, Pavlides S, Llaverias G, Park DS, Lisanti MP: Caveolin-1 and regulation of cellular cholesterol homeostasis. Am J Physiol Heart Circ Physiol 2006, 291:H677-H686

12. Razani B, Combs TP, Wang XB, Frank PG, Park DS, Russell RG, Li M, Tang B, Jelicks LA, Scherer PE, Lisanti MP: Caveolin-1-deficient mice are lean, resistant to diet-induced obesity, and show hypertriglyceridemia with adipocyte abnormalities. J Biol Chem 2002, 277:8635-8647

13. Hassan GS, Jasmin JF, Schubert W, Frank PG, Lisanti MP: Caveolin-1 deficiency stimulates neointima formation during vascular injury. Biochemistry 2004, 43:8312-8321

14. Schubert W, Frank PG, Woodman SE, Hyogo H, Cohen DE, Chow
CW, Lisanti MP: Microvascular hyperpermeability in caveolin-1(-/-) knock-out mice. Treatment with a specific nitric-oxide synthase inhibitor, L-NAME, restores normal microvascular permeability in Cav-1 null mice. J Biol Chem 2002, 277:40091-40098

15. Chidlow JH Jr, Greer JJ, Anthoni C, Bernatchez P, FernandezHernando C, Bruce M, Abdelbaqi M, Shukla D, Granger DN, Sessa WC, Kevil CG: Endothelial caveolin-1 regulates pathologic angiogenesis in a mouse model of colitis. Gastroenterology 2009, 136:575-584

16. Dewever J, Frerart F, Bouzin C, Baudelet C, Ansiaux R, Sonveaux P, Gallez B, Dessy C, Feron O: Caveolin-1 is critical for the maturation of tumor blood vessels through the regulation of both endothelial tube formation and mural cell recruitment. Am J Pathol 2007, 171:1619-1628

17. Lin MI, Sessa WC: Antiangiogenic therapy: creating a unique "window" of opportunity. Cancer Cell 2004, 6:529-531

18. Fernández-Hernando $C, Y u$ J, Suarez $Y$, Rahner C, Davalos A Lasuncion MA, Sessa WC: Genetic evidence supporting a critical role of endothelial caveolin-1 during the progression of atherosclerosis. Cell Metab 2009, 10:48-54

19. Frank PG, Lee H, Park DS, Tandon NN, Scherer PE, Lisanti MP: Genetic ablation of caveolin-1 confers protection against atherosclerosis. Arterioscler Thromb Vasc Biol 2004, 24:98-105

20. Yu J, Bergaya S, Murata T, Alp IF, Bauer MP, Lin MI, Drab M, Kurzchalia TV, Stan RV, Sessa WC: Direct evidence for the role of caveolin-1 and caveolae in mechanotransduction and remodeling of blood vessels. J Clin Invest 2006, 116:1284-1291

21. Fulton D, Babbitt R, Zoellner S, Fontana J, Acevedo L, McCabe TJ Iwakiri Y, Sessa WC: Targeting of endothelial nitric-oxide synthase to the cytoplasmic face of the Golgi complex or plasma membrane regulates Akt- versus calcium-dependent mechanisms for nitric oxide release. J Biol Chem 2004, 279:30349-30357

22. Bauer PM, Yu J, Chen Y, Hickey R, Bernatchez PN, Looft-Wilson R, Huang Y, Giordano F, Stan RV, Sessa WC: Endothelial-specific expression of caveolin-1 impairs microvascular permeability and angiogenesis. Proc Natl Acad Sci USA 2005, 102:204-209

23. Kuhlencordt PJ, Gyurko R, Han F, Scherrer-Crosbie M, Aretz TH, Hajjar R, Picard MH, Huang PL: Accelerated atherosclerosis, aortic aneurysm formation, and ischemic heart disease in apolipoprotein E/endothelial nitric oxide synthase double-knockout mice. Circulation 2001, 104:448-454

24. Garcia-Cardeña G, Fan R, Stern DF, Liu J, Sessa WC: Endothelial nitric oxide synthase is regulated by tyrosine phosphorylation and interacts with caveolin-1. J Biol Chem 1996, 271:27237-27240

25. Frank PG, Lisanti MP: Caveolin-1 and caveolae in atherosclerosis: differential roles in fatty streak formation and neointimal hyperplasia. Curr Opin Lipidol 2004, 15:523-529

26. Galkina E, Ley K: Vascular adhesion molecules in atherosclerosis Arterioscler Thromb Vasc Biol 2007, 27:2292-2301

27. Kawashima S, Yamashita T, Ozaki M, Ohashi Y, Azumi H, Inoue N, Hirata K, Hayashi Y, Itoh H, Yokoyama M: Endothelial NO synthase overexpression inhibits lesion formation in mouse model of vascular remodeling. Arterioscler Thromb Vasc Biol 2001, 21:201-207

28. Cerezo A, Guadamillas MC, Goetz JG, Sánchez-Perales S, Klein E Assoian RK, del Pozo MA: The absence of caveolin-1 increases proliferation and anchorage-independent growth by a Rac-dependent, Erk-independent mechanism. Mol Cell Biol 2009, 29:50465059 